\title{
VARIABLES DETERMINANTES PARA UNA NUEVA CULTURA TUTORIAL DESDE EL PAT
}

\author{
Asunción Lledó Carreres \\ asuncion.lledo@gcloud.ua.es \\ Gonzalo Lorenzo Lledó \\ glledo@ua.es \\ Marcos Gómez Puerta \\ marcos.gomez@ua.es \\ Alejandro Lorenzo Lledó \\ alejandro.Iorenzo@ua.es \\ Facultad de Educación \\ Universidad de Alicante
}

Fecha de Recepción: 1 Marzo 2018

Fecha de Admisión: 10 Abril 2018

\section{RESUMEN}

Las universidades españolas ha asumido el reto de implementar procesos de acompañamiento tutorial desde programas institucionales de acción tutorial (PAT) consideradas como actuaciones programadas para la inclusión del alumnado de nuevo ingreso en la universidad con la finalidad de cubrir sus necesidades en cuanto a información, formación y asesoramiento continuado. El objetivo de este estudio ha sido identificar e implementar acciones tutoriales a partir de variables determinantes para un cambio de dirección en las acciones tutoriales del PAT, como son: coordinación en función de los agentes implicados; Equipo docente del PAT y alumnado tutorizado. En este estudio y desde una perspectiva ideográfica y cualitativa se ha utilizado el estudio de caso de carácter descriptivo. Los informes realizados por los participantes en cada una de las dimensiones: a nivel de coordinación general, a nivel de equipo docente y a nivel de alumnado tutorizado, han sido utilizados para la recogida de la información requerida. Los resultados obtenidos muestran a partir de las variables establecidas unas acciones tutoriales que no solo contemplan el apoyo al estudiante en el ámbito académico sino en gran parte supone una orientación hacia el acompañamiento tutorial en aspectos personales y obstáculos que se pueden suceder a lo largo de la trayectoria del estudiante universitario por lo que se concluye seguir avanzando en este modelo de acción tutorial a través del PAT.

Palabras clave: innovación; acompañamiento tutorial; PAT; apoyo personal 
ABSTRACT

Determining variables for a new tutorial culture from the PAT

Spanish universities have taken on the challenge of implementing tutorial accompaniment processes from institutional tutorial action programmes (PAT) considered as programmed actions for the inclusion of new students in the university with the aim of covering their needs in terms of information, training and ongoing advice. The objective of this study has been to identify and implement tutorial actions based on determining variables for a change of direction in the TAP tutorial actions, such as: coordination according to the agents involved; TAP teaching team and tutoring students. $\mathrm{n}$ this study and from an ideographic and qualitative perspective, the descriptive case study has been used. The reports made by the participants in each of the dimensions: at the level of general coordination, at the level of the teaching team and at the level of the tutored students, have been used to collect the required information. The results obtained show that, based on the variables established, tutorial actions not only contemplate support for the student in the academic sphere, but also, to a large extent, imply an orientation towards tutorial accompaniment in personal aspects and obstacles that may occur throughout the university student's career, which is why it is concluded that this tutorial action model will continue to advance through the PAT.

Keywords: innovation; tutorial support; PAT; personal support

\section{ANTECEDENTES}

La calidad de la educación se ha convertido en una cuestión elemental y prioritaria en nuestro entorno (Unión Europea). Una institución educativa de calidad ha de ofertar lo que sus usuarios necesitan: hacer bien aquello que se tiene que hacer (Rodríguez Espinar, 2002, 2006). En este sentido, desde el contexto del EEES se ha dado una cierta prioridad a los procesos de asesoramiento y acompañamiento tutorial como una herramienta clave para mejorar la calidad educativa al mismo tiempo que con ello se contribuye al cambio de una nueva cultura docente, no únicamente centrada en la transmisión de contenidos sino que se da paso a una paradigma educativo centrado en los procesos de aprendizaje de los estudiantes universitarios desde una atención más personalizada y comprensiva que haga posible la asunción de la diversidad y la multiculturalidad existentes en las aulas universitarias.

Desde el paradigma educativo generado desde el marco europeo se ha acrecentado la priorización de una formación conformada alrededor de la adquisición de competencias y un proceso de aprendizaje que da paso al protagonismo del trabajo autónomo del estudiante universitario. Ello va a requerir una tutela en el proceso de aprendizaje que anteriormente apenas tenía presencia en el contexto universitario. Ya en el Informe Universidad Bricall (2000) se apostaba por un nuevo rol de tutorización del docente universitario que abarcaría las siguientes áreas de actuación: el asesoramiento previo al ingreso en la Universidad, en la planificación de los estudios, en apoyos especiales, en casos de crisis o dificultades particulares de algún estudiante y el asesoramiento y apoyo al desenvolvimiento formativo de los estudiantes. Es decir, a propuestas encaminadas a la atención de necesidades individuales de información, formación y orientación en su diseño curricular y profesional (Lledó, 2014).

El nuevo rol docente desde la acción tutorial convierte al profesorado universitario como tutor y profesor/a de referencia del grupo de alumnos y alumnas que va a tutelar y como apunta Lázaro (2002; 2008) tutorizará la formación, tanto humana como científica, de un estudiante en concreto, al que también acompaña en todo su proceso de aprendizaje, definiendo la acción tutorial como "una competencia educativa inmersa en la perspectiva orientadora o de asesoramiento del aprendizaje, no solo instructivo, que todo educador ha de ejercer como tutela del proceso formativo de acompañamiento" (Lázaro, 2008, 119). En este sentido aunque ya partimos de un modelo de corte tra- 
dicional y marcadamente instructivo, restringido casi exclusivamente a resolver las dudas de las asignaturas y caminamos hacia una tutoría más centrada en el acompañamiento y asesoramiento al comienzo y a lo largo de la carrera universitaria del estudiante universitario. Apostando por esta nueva perspectiva, la tutoría universitaria puede contribuir a una formación más integral del estudiante universitario (Luna, 2012; Rodríguez Sierra, 2012; Vázquez y Cuevas, 2014; Álvarez y Álvarez, 2013, 2015) así como una acción nuclear que ofrezca una formación integral y de calidad, que atienda a las peculiaridades de un alumnado heterogéneo en el actual contexto universitario (Pérez, González, González y Martínez, 2017) y convierta a la acción tutorial como una actividad inherente a la función docente.

Como consecuencia de dicho marco universitario las universidades españolas ha asumido el reto de implementar procesos de acompañamiento tutorial desde programas institucionales. Desde el año 2005/2006 hasta la actualidad, se están implementando en la Universidad de Alicante programas institucionales de acción tutorial (PAT) consideradas como actuaciones programadas para la inclusión del alumnado de nuevo ingreso en la universidad con la finalidad de cubrir sus necesidades en cuanto a información, formación y asesoramiento continuado. Desde los mismos, ya en sus comienzos se pretendían los siguientes objetivos:

Orientar al alumnado de primero y de nuevo ingreso en el conocimiento de la Universidad para una mayor integración en el nuevo contexto universitario.

Informar al alumnado tutorizado sobre cuestiones académicas y /o profesionales.

Fomentar la participación del alumnado en los distintos ámbitos de la vida universitaria.

Reflexionar sobre el desarrollo académico y personal del alumno autorizado en relación a los estudios iniciados.

Valorar la necesidad del apoyo tutorial como instrumento de conocimiento y reflexión en el proceso de formación universitaria.

Asimismo, se ha planificado intervenir en una serie de áreas de trabajo a lo largo de la implementación del proceso tutorial:

Adaptación al contexto curricular y social universitario.

Desarrollo académico y trayectoria en general.

Desarrollo de un aprendizaje eficaz.

Diagnóstico de dificultades académicas y personales.

Conocimiento de los puntos débiles y fuertes en su proceso de estudio.

Conocimiento de cómo aprende el alumno/a y su nivel de compromiso.

Búsqueda de apoyos en su aprendizaje.

Conocimiento de las expectativas personales y profesionales del alumno/a.

Evaluación de sus logros.

\section{OBJETIVOS}

Sin embargo con el trabajo implementado a lo largo de más de una década se han constatado disfunciones que están poniendo en tela de juicio la efectividad de las acciones tutoriales universitarias. Es por ello, que este trabajo tiene como problema de investigación analizar el estado actual del PAT y en base a ello, como objetivo general identificar e implementar acciones tutoriales a partir de variables determinantes para un cambio de dirección en las acciones tutoriales del PAT. A partir del mismo se han planteado unos objetivos más específicos en el estudio empírico y que han sido los siguientes:

Crear un marco institucional que planifique, coordine y supervise el PAT.

Establecer unas directrices generales como marco de actuación para todos los centros 
(Facultades y Escuela) a la vez que se faciliten orientaciones flexibles que puedan ser adaptadas a cada contexto.

Dar respuesta a las necesidades de acompañamiento y orientación del estudiante en su adaptación al contexto universitario y en el desarrollo académico, personal y profesional.

A partir de los objetivos planteados, se han marcado una serie de dimensiones que servirán para identificar variables determinantes en este proceso de una nueva dirección del PAT y que han sido las siguientes:

Contexto institucional.

Coordinación Facultades

Equipo de Tutores y Tutoras de las Facultades.

Alumnado Tutor/Tutora

Alumnado tutorizado

Asimismo, las variables del estudio en función de las dimensiones establecidas han sido:

Variable 1. Coordinación en función de los agentes implicados: coordinación general del PAT y coordinación desde las respectivas Facultades.

Variable 2. Equipo docente del PAT.

Variable 3. Alumnado tutorizado.

\section{MUESTRA DE PARTICIPANTES}

La muestra de participantes se ha conformado por: 9 coordinadores/as generales; 142 Tutores y Tutoras de las Facultades, 33 de alumnado tutor/a y 4420 de alumnado tutorizado.

\section{MÉTODOLOGÍA E INSTRUMENTOS}

\subsection{Método}

Desde una perspectiva ideográfica y cualitativa se ha utilizado, en una parte de la investigación que se presenta, el estudio de caso de carácter descriptivo, que como indican Quintana y García (2012) sirve para conocer e identificar aquellas variables más significativas de un hecho o realidad social, en nuestro caso desde las acciones tutoriales implementadas a través del PAT en la Universidad de Alicante.

\subsection{Instrumento}

En cuanto a la parte del estudio referida al estudio de caso se ha recogido la información a través de informes realizados por el profesorado coordinador de las respectivas facultades como los realizados por los propios profesores tutores y tutoras de los distintos grupos de alumnado tutorizado. Dichos informes recogían los siguientes ámbitos en función de las dimensiones del estudio:

A nivel de coordinación general.

A nivel de equipo docente tutor/a

A nivel de alumnado tutor/a

A nivel de alumnado tutorizado.

\section{RESULTADOS}

Los resultados se presentan en función del objetivo del estudio y las dimensiones establecidas y las variables del estudio:

Variable 1. Coordinación en función de los agentes implicados: coordinación general y coordinación en las respectivas Facultades

En la tabla 1 se presentan los resultados referidos a la dimensión contexto institucional: 
Tabla 1

Acciones tutoriales implementadas desde el contexto institucional

\begin{tabular}{|c|c|}
\hline A nivel de coordinación general & Acciones tutoriales \\
\hline - Difusión y conocimiento del PAT & $\begin{array}{l}\text {-Presencia del PAT en la actividad general de } \\
\text { conocimiento de la universidad estudiantes de } \\
\text { secundaria. } \\
\text {-Información durante el proceso de matriculación. }\end{array}$ \\
\hline - Marco institucional & $\begin{array}{l}\text {-Comisión del PAT: tareas de gestión y coordinación } \\
\text { de la acción tutorial. }\end{array}$ \\
\hline - Coordinación general & $\begin{array}{l}\text {-Guía PAT: objetivos del PAT, cronograma, } \\
\text { funciones y tareas realizar. } \\
\text {-Sesiones de trabajo grupales y colaborativas de los } \\
\text { coordinadores de las Facultades y la coordinadora } \\
\text { general del PAT. }\end{array}$ \\
\hline -Coordinación general en las Facultades & $\begin{array}{l}\text {-Jornadas de acogida alumnado nuevo ingreso. } \\
\text {-Difusión del PAT a nivel de Facultad. } \\
\text {-Constitución del equipo docente de profesorado } \\
\text { tutor/a. } \\
\text {-Trabajo Guía PAT y planificación de sesiones } \\
\text { grupales con el alumnado tutorizado. }\end{array}$ \\
\hline - Necesidades & $\begin{array}{l}\text {-Sesiones de trabajo grupales para unificar criterios } \\
\text { de actuación. }\end{array}$ \\
\hline
\end{tabular}

Como se indica, se muestran las acciones tutoriales implementadas desde la Variable 1. Coordinación en función de los agentes implicados. Se constata toda una serie de actuaciones de coordinación en la que participan los agentes implicados en la coordinación general tanto desde el Instituto de Ciencias de la Educación (ICE) como desde las respectivas Facultades.

Variable 2. Equipo docente del PAT.

Los resultados referidos a las acciones tutoriales implementadas referidas a la dimensión coordinación por parte del equipo docente tutor se presentan en la tabla 2: 


\section{VARIABLES DETERMINANTES PARA UNA NUEVA CULTURA TUTORIAL DESDE EL PAT}

Tabla 2

Acciones tutoriales implementadas desde el equipo docente tutor/a y alumnado tutor/tutora

\begin{tabular}{|c|c|}
\hline A nivel del equipo docente tutor/tutora & Acciones tutoriales \\
\hline - Coordinación & $\begin{array}{l}\text {-Asistencia a sesiones grupales para coordinación } \\
\text { general del PAT de la Facultad correspondiente. } \\
\text {-Sesiones colaborativas entre el profesorado tutor/a } \\
\text { para detectar y analizar los problemas de ámbito } \\
\text { curricular, personal o social que dificultan el } \\
\text { aprendizaje. }\end{array}$ \\
\hline - $\quad$ Sesiones tutoriales & $\begin{array}{l}\text {-Planificación de las sesiones con el grupo de } \\
\text { alumnado a tutorizar. } \\
\text {-Sesión grupal de conocimiento del grupo a tutorizar } \\
\text { y sus necesidades. } \\
\text {-Planificación de las actividades de acompañamiento } \\
\text { a realizar con el grupo tutorizado en función de las } \\
\text { demandas y necesidades. } \\
\text { - Actividades grupales de acompañamiento y } \\
\text { colaborativas por parte del tutor/tutora con el grupo } \\
\text { tutorizado. } \\
\text {-Asistencia a actividades formativas. } \\
\text {-Realización de tutorías virtuales e individuales } \\
\text { presenciales. }\end{array}$ \\
\hline - $\quad$ Necesidades & $\begin{array}{l}\text {-Información de temas administrativos. } \\
\text { - Información sobre temas académicos referidos a } \\
\text { becas, movilidad, itinerarios, máster, idiomas... } \\
\text {-Talleres formativos en estrategias de tutorización y } \\
\text { dinámicas de grupo. } \\
\text {-Taller formativo sobre adaptaciones curriculares en } \\
\text { el contexto universitario. } \\
\text {-Jornadas de convivencia y formativas en PAT. }\end{array}$ \\
\hline
\end{tabular}

Como se indican en la tabla 2, se muestran las acciones tutoriales programadas y que han sido implementadas desde la Variable 2. Equipo docente del PAT en las que se evidencian acciones tutoriales de acompañamiento y colaborativas tanto por parte del equipo docente del profesorado como del alumnado tutor/tutora.

Variable 3. Alumnado tutorizado.

Los resultados referidos a las acciones tutoriales planificadas e implementadas en el alumnado tutorizado se muestran en la tabla 3 : 
Tabla 3

Acciones tutoriales implementadas en el alumnado tutorizado

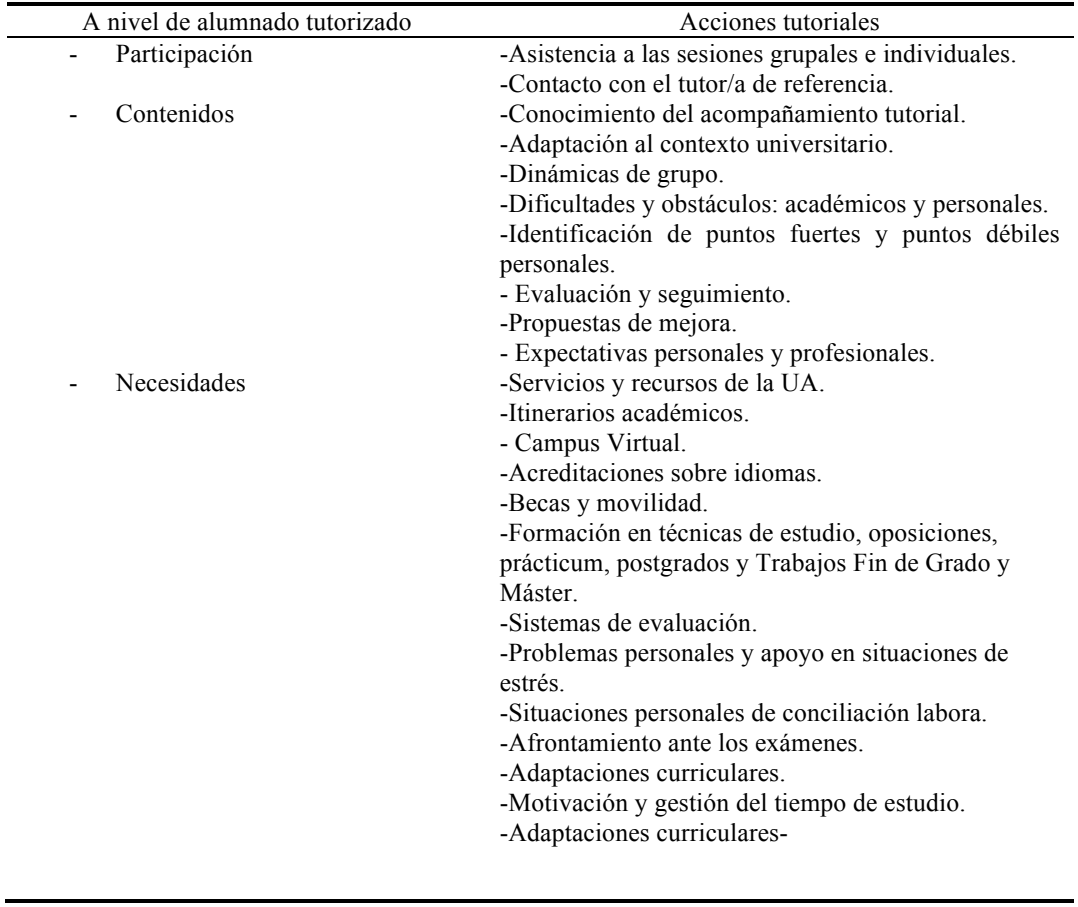

En los resultados mostrados en la tabla 3, en relación a la variable alumnado tutorizado se presentan tantos acciones tutoriales referidas a aspectos académicos como personales. En el mismo sentido también se aprecian necesidades demandadas por el alumnado tutorizado tanto académicas como personales.

\section{DISCUSIÓN Y CONCLUSIONES}

En función del objetivo del estudio y en relación con estudios previos (Lázaro, 2008; Álvarez González, 2013; Álvarez y Álvarez, 2015; Pérez, González, González y Martínez, 2017) nuestros resultados marcan una nueva orientación a las acciones tutoriales que desde el marco institucional de la Universidad de Alicante se está coordinando desde el Programa de acción Tutorial (PAT) y gestionado y coordinado por el Vicerrectorado de Calidad e Innovación Educativa y El Instituto de Ciencias de la Educación, que coordinada la primera autora de dicha comunicación.

En este sentido, de un modelo tradicional de tutoría limitado exclusivamente a lo académico, iniciamos una nueva dirección que supone una nueva cultura tutorial, orientada a una relación de ayuda y acompañamiento desde la acogida al nuevo contexto universitario, al desarrollo de su itinerario académico, estando presente en los obstáculos y dificultades que puedan sucederse hasta la orientación en vías a su futuro profesional.

Como se muestran en nuestros resultados implementados hay un trabajo coordinado y colaborativo por parte de los agentes implicados (contexto institucional y equipo docente) que pretende cubrir en gran medida las necesidades demandadas por el alumnado participante en el PAT. 
Concluimos a partir de los resultados presentados y en función de las acciones tutoriales implementadas, las variables determinantes para una nueva cultura del PAT:

Variable 1. Coordinación en función de los agentes implicados:

Necesidad de una coordinación general que unifique criterios de actuación y que cubra las necesidades formativas del equipo docente del PAT en su nuevo rol tutorial.

Publicación de una Guía del PAT a nivel general que coordine las acciones tutoriales en el contexto universitario.

Una mayor difusión del PAT entre el alumnado en las jornadas de bienvenida de las respectivas Facultades.

Realización de talleres formativos para el equipo docente del PAT sobre una formación más psicopedagógica de la acción tutorial, incidiendo en el desarrollo personal, interpersonal y profesional. Variable 2. Equipo docente del PAT.

Implicación del equipo docente en las tareas tutoriales.

Trabajo colaborativo entre el profesorado participante en el PAT mediante redes y comunidades de aprendizaje.

Utilización de recursos TIC como herramienta de difusión y comunicación.

Estudio y seguimientos de las necesidades del estudiante universitario en función de las distintas titulaciones y situaciones personales.

Utilización de la tutoría entre iguales en el equipo docente del PAT.

Variable 3. Alumnado tutorizado

Planificación de tutorías presenciales grupales cooperativas entre el alumnado así como tutoría individuales presenciales y virtuales a demanda del alumnado.

Concienciación y difusión del PAT y los beneficios que aporta al estudiante universitario.

Ubicación de una franja horaria en el horario académico del estudiante para facilitar su asistencia a las sesiones grupales programadas dentro del PAT.

Utilización de estrategias en las sesiones grupales que favorezcan el conocimiento del grupo y un clima óptimo para la comunicación y desarrollo personal e interpersonal hacia una tutoría más integral del proceso formativo del estudiante universitario.

Evaluación del grado de satisfacción del estudiante universitario participante en el PAT. Con relación a esta variable es la segunda parte de este estudio.

Para finalizar, consideramos que hay que valorar a partir de los estudios como el que se presenta, la necesidad del apoyo tutorial como instrumento de conocimiento y reflexión en el proceso de formación universitaria desde todos los ámbitos y desde una visión más integral y global del desarrollo humano.

\section{REFERENCIAS BIBLIOGRÁFICAS}

Álvarez González, M. (2013). Hacia un modelo integral de la tutoría universitaria. En M. Álvarez González y R. Bisquerra (Coords), Manual de orientación y tutoría. Barcelona: Wolters Kluwer España.

Álvarez Pérez, P., González, M. C. (2009). Modelo comprensivo para la institucionalización de la orientación y la tutoría en la enseñanza universitaria. Revista Qurriculum, 22, 73-95.

Álvarez, M. \& Álvarez, J. (2015). La tutoría universitaria: del modelo actual a un modelo integral. Revista Electrónica Interuniversitaria de Formación del Profesorado, 18 (2), 125-142.

Bricall, Josep M. (2000): Informe Universidad 2000. Barcelona: Conferencia de Rectores de las Universidades Españolas (CRUE).

Gairín, J. y otros (2008). Estudi per analitzar dades i causes de l'abandonament dels estudiants a la 
universitat. Barcelona: Agència per la Qualitat Universitària (AQU) de Catalunya.

González Palacios, A., y Avelino Rubio, I. (2016). Tutoría: Una revisión conceptual. Revista de Educación y Desarrollo, 38, 57-68.

Lázaro, A (2002) La acción tutorial de la función docente universitaria. En Álvarez Rojo y A. Lázaro (Coords.) Calidad de las Universidades y Orientación universitaria. Málaga: Aljibe.

Lázaro, A. J. (2008). Diferencias cualitativas entre experiencias tutoriales para opciones de aprendizaje universitario. Revista Interuniversitaria de Formación del Profesorado, 61, pp. 109-138.

Luna, C. (2012). Fortalezas, debilidades y propuestas de mejora del Plan Institucional de Orientación y Tutoría del Instituto Tecnológico de Celaya. En AA.VV., Actas de Seminario Hacia un Nuevo Plan Integral de Orientación y tutoría. México: Instituto Tecnológico de Celaya.

Pérez, F.J, González, Cr., González, N. y Martínez, M. (2017). Tutoría en la Universidad: un estudio de caso en la Facultad de Educación de la Universidad de Murcia. Educatio Siglo XXI, Vol. 35 no $2 \cdot 2017$, pp. $91-110$

Pérez, J., Martínez, P., y Martínez, M. (2015). Satisfacción del estudiante universitario con la tutoría. Diseño y validación de un instrumento de medida. Estudios sobre Educación, 29, 81-101.

Quintana, J., García, B., (coords) (2012). Fundamentos básicos de metodología de investigación educativa. Madrid: CCS.

Rodríguez Espinar, S. (2002). La orientación y la función tutorial, factores de la calidad de la educación. En CES (Ed.). Los educadores en la sociedad del S. XXI (pp. 89-160). Madrid: MEC.

Rodríguez Espinar, S. (2006). Función tutorial y calidad de la educación. En M. Álvarez González (Coord.). La acción tutorial: su concepción y su práctica (pp. 9-25). Madrid: MEC.

Rodríguez Sierra, A. V. (2012). Análisis contextual de la acción tutorial en la corporación universitaria Minuto de Dios (Bogota, Colombia) para la elaboración de un modelo organizativo y de intervención (Tesis Doctoral no publicada) Universidad de Valladolid.

Vázquez, F. \& Cuevas. (2014). Experiencia de análisis de necesidades de la acción tutorial en la Universidad de Guanajuato. En M. Álvarez González y R. Bisquerra (Coords), Manual de orientación y tutoría. Barcelona: Wolters Kluwer España. 
